\title{
Study of wildfire in-draft flows for counter fire operations
}

\author{
R. Roxburgh \& G. Rein \\ BRE Centre for Fire Safety Engineering, University of Edinburgh, UK
}

\begin{abstract}
The use of counter-fires to gain control over wildfires is a technique used by some fire services around the world. A fire is purposely lighten ahead of the wildfire and the buoyancy induced in-drafts pull it towards the flame front thus creating a fire break of burnt fuel. Well used, this technique is fast, effective and safe. However, no technical research has been done on the subject. Without understanding of the mechanisms, counter-firing remains a difficult technique and can lead to unnecessary risks being taken or opportunities lost. This paper uses computational fluid dynamics to study the in-draft created around wildfires. A generalized structure of the in-draft velocity profile composed of three zones is observed. The length of the different zones are analysed to find the dependence with fire intensity and wind velocity.
\end{abstract}

Keywords: entrainment, fire fighting, suppression, back fire, computer modelling, CFD, FDS.

\section{Introduction}

A common method of wildfire suppression is the use of fire breaks to stop the progress of a flaming front. Fire breaks are strips of land from which all or most of the flammable materials have been removed [1-3]. These strips create fuelless barriers past which the wildfire cannot spread as it no longer has fuel. These can often be incorporated into natural fire barriers such as rivers, canyons and roads in order to increase their size and effectiveness. The width of a fire break has to be sufficient large to avoid the flaming ignition across it. A common rule based on the radiant power states that a fire-break must be one and a half times as wide as the flame height $[2,3]$. One speedy way of creating a fire break is to light a counter fire a certain distance in front of the main wildfire. Due to the 
buoyant-induce flows of the wildfire plume, air is entrained into the flame causing local in-draft winds around the flame. If the counter-fire is set at some distance from the wildfire in-draft will pull the counter-fire towards the main fire, just as atmospheric winds drive wildfires. When these two fires meet, the counter-fire will have burnt the fuel behind it and thus will have created a firebreak of some distance [3]. Practitioners assert that well used, this technique is fast, effective and safe. The main problem with using this technique is the uncertainty over the distance from the main wildfire where the counter fire should be lit. If it is placed too far away where in-draft is low, it will not be pull towards the wildfire but be dominated by atmospherics wind conditions and could start a secondary fire propagating ahead of the main front. It if is placed too close to the wildfire, the width of the fire break created will not be enough to stop the spreading. The technical literature contains very poor information on this fire-fighting technique. No experimental or theoretical study has been found. Only a few firefighting manuals [5] provide limited practical information. To the best knowledge of the authors, no previous work has been carried out to study the flow dynamics of counter-fires. This paper is an original investigation into the use of CFD to study the entrainment flows around a wildfire with the intention of providing estimates on the optimum range of distance where to lit counter fire.

\section{Fire driven computational fluid dynamics}

The simulations conducted here used Fire Dynamic Simulator 5 (FDS5) [6]. FDS5 is a LES computational fluid dynamic model intended for fluid flows driven by fire. This program was chosen for this project because of its ability to model both fluid flow and fires. LES is used as it can be applied to cells which are very large and approximate the transport of the very small, turbulent eddies but averaging them over space and not time. This allows simulating the movement of the larger eddies (in the order of $5 \mathrm{~cm}$ ). As this report looks into the large scale flow patterns in the entrainment of a wildfire it felt that using the LES model to approximate small scale eddies is a valid assumption as it is not these eddies we are as interested in.

\section{Scenario}

The results obtained within this report are gained through the use of a twodimensional simulation model. The domain, shown in Fig. 1, contains a fire of some size on a flat ground with wind coming from the right side. It spans horizontally downstream of the wildfire to include the region where the in-draft is generated and spans vertically to include the fire plume. This is a first approximation to the problem, and thus the domain has been kept simple with no trees or vegetation, topography or inclination of the terrain. It is a flat terrain with no obstacles to the flow. The counter-fire is not included in the simulations since it is initially a small fire compared to the approaching wildfire and thus its effects on the flow field can be neglected during the ignition stage. 


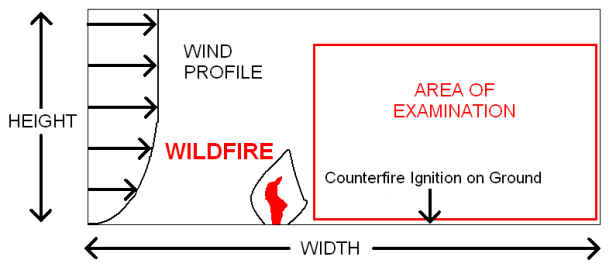

Figure 1: The basic scenario under study for counter firing conditions.

The problem is treated as two dimensional and the wildfire as a non-spreading source of fixed intensity. By fixing the wildfire in one place the assumption of quasi-stationary flows is invoked because wildfire spread at significantly lower velocities than the flow around them. The two-dimensional representation allows for a reduction in the computational cost by two orders of magnitude. A recent VTT study [4] compares the results of two and three dimensional models of wildfires. The conclusions validate the application of two-dimensional analysis to this study since the entrainment flow that is being analyzed here is more concerned with flow patterns and range of values rather than finely accurate velocities fields.

\subsection{Steady state analysis}

Wildfires propagate at a significantly lower velocity that the transport of heat and momentum by the plume. Thus, it can be assumed a quasi-steady state process. . This means that the wildfire releases heat at a constant average rate over their propagation and that the characteristic properties of the fire entrainment, such as temperature and velocity fields fluctuate but reach a steady regime after some time.. In the simulations, ignition is started at the initial time and the fire reaches the peak heat release rate within a few seconds. The time it takes for the surrounding flow to react to the change in conditions is monitored to assure steady-state conditions are reached. The analysis concluded that the simulations in the range of conditions under studied take at least $15 \mathrm{~s}$ to reach steady state. Once in these conditions, the results are averaged over a further $45 \mathrm{~s}$ of simulation to assure than the fluctuating nature of the fire environment is taken into account.

\subsection{Boundary conditions}

The model was set up with four boundaries; the bottom edge being designated the ground, and the top and side of the domain being open to the atmosphere. The open boundary conditions allow air to pass into and out of the domain as would be possible in real life. A wind profile was applied to the upstream right boundary to create an atmosphere wind profile (taken from [4]).

\subsection{Boundary and grid size analysis}

The location of the boundaries in a open-domain problem is an important part of the modeling process. If the domain is made too small, the boundary conditions 
will affect the results which are obtained and will reduce their accuracy. If, however, the domain is made too large, valuable computer time will be wasted simulating processes which have no impact on the solution being investigated. A boundary-location study was therefore carried out to find the optimum domain size for the problem. It was found that the domain should be approximately $90 \mathrm{~m}$ in width and $28 \mathrm{~m}$ in height, with the fire located $20 \mathrm{~m}$ from the wind boundary (left side in Fig 1). Grid is also important as the size of each cell within the domain affect the accuracy of the mathematical solution. While smaller cells could be more accurate and provide higher resolution in predicting the flow than large cells, they also require longer computer times to simulate. A practical compromise solution must be met. The accuracy criteria used was taken from the guidelines given in [6] based on a minimum resolution for the fire plume diameter. After a study was carried out it was found that each cell should be 15 $\mathrm{cm}$ by $15 \mathrm{~cm}$ in size in order to maximise the accuracy of the simulations within the time restraints for this investigation.

\subsection{Validation}

As previously mentioned, FDS has noted been validated for open-air fires and so a series of simulations were conducted to test the tool in this context. The induced-flow predicted by FDS cannot be validated in detail for this application since suitable theory or experimental measurements have not been found. Thus, the validation is done using simple plume predictions in open domains. The first series of tests compared the flame height observed in the simulations to a theoretical flame height found using Heskestad's correlation [7]. It was found that for all the intensities of fires used the predicted flame height was within $10 \%$ of this theoretical value. The second series of test compared temperatures within the fire plume to theoretical values obtained from [7]. Temperatures at various points along the centerline of the plume were compared and it was found that the simulated model was always within $15 \%$ of the theoretical model for the range of conditions under study.

\section{Results and analysis}

A wide range of conditions pertaining to real wildfire situations were studied. The fire intensities range from 1 to $10 \mathrm{MWm}^{-1}$ and wind velocities range from 1 to $15 \mathrm{~ms}^{-1}$. These ranges cover from small to large wildfires and from stagnant to strong wind conditions [4]. In order to study the entrainment flow field that is of interest when setting counter fires, the results at a fixed set of points within the domain were monitored. These points were located at five different heights $(0.25 \mathrm{~m}, 0.5 \mathrm{~m}, 0.75 \mathrm{~m}, 1 \mathrm{~m}$ and $2 \mathrm{~m})$ from the ground and at $2 \mathrm{~m}$ intervals from the edge of the fire towards the downstream side (right) of the domain. This allows studying a horizontal profile of in-draft velocities at different distances from the fire. The heights of the monitoring points were kept as low as this would be the zone in which the counter fire would be set. The point velocities were recorded at much faster rate than the frequency of the flow fluctuations. 
As well as in-draft horizontal profiles, FDS5 provided transient 3D flow fields. These were used to conclude on the overall pattern fluid dynamics and analyzed for special features like characteristic eddies and the motion of large vortexes.

\subsection{General horizontal profiles}

The point velocities obtained from each simulation were analyzed as the average steady-state horizontal velocity. Inspection of the resulting profiles of velocity vs. distance indicate the existence of characteristic flow regions. Figure 2 shows this general profile and differentiates six lengths describing three flow regions the in-draft profile of any simulation. These regions are refereed to as Zones 1, 2 and 3. Zone 1 of a total length $\mathrm{L}_{1}$, is the closet to the fire and affected by the tilted flame. This zone can be separated into the wind affected length $\mathrm{L}_{\mathrm{w}}$ and the wind-independent length $\mathrm{L}_{0}$. Zone 2 of total length $\mathrm{L}_{2}$, is where the peak velocity $\mathrm{U}_{\mathrm{P}}$ at distance Lp. Zone 3 starts at $\mathrm{L}_{\mathrm{d}}$ and is the area where atmospheric conditions dominate and the fire in-draft is no longer felt.

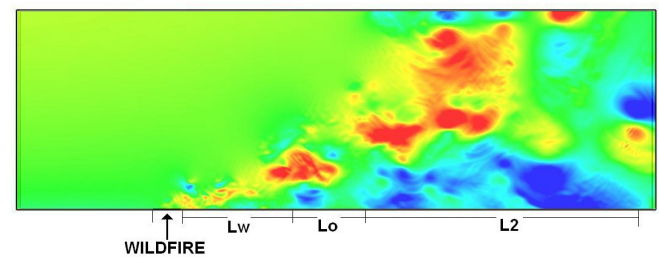

Figure 2: Typical horizontal velocity field obtained from FDS5. Red is positive and blue negative $\mathrm{x}$-velocities.

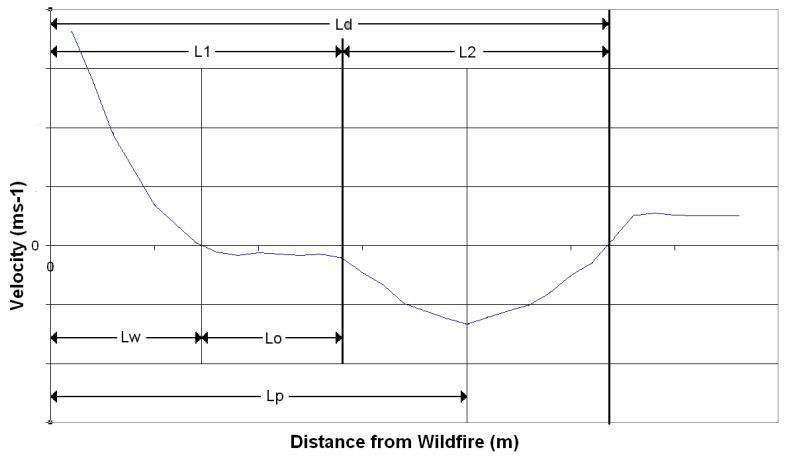

Figure 3: Horizontal velocity near the ground and ahead of the wildfire front.

The flame-affected zone is not ideal for the setting of counter-fires as it contains adverse in-draft velocities and is too close to the wildfire. Thus a counter fire in this region would not create a fire break of sufficient width. The results state that close to the flame the horizontal in-draft component velocity is 
the lowest or even pushes the counter fire away. This zone can be split into the wind dependent $\mathrm{L}_{\mathrm{w}}$ and a the wind-independent $\mathrm{L}_{0}$. The wind dependent length is only found in simulations that have high winds and the steady-state horizontal velocity is positive and thus pushing the counter-fire away from the wildfire instead of pulling it. The reason for this is that the wind causes the flame to be blown over this zone. $\mathrm{L}_{0}$ is the length of the zone with a negative horizontal velocity affected by close presence of the plume above it. The plume creates a recirculation patterns that causes reduced horizontal velocities in this zone. The peak-in-draft zone of the profile has the largest horizontal velocity but also has an area either side of this peak in which the counter-fire would be drawn into the main wildfire. This is the zone in where fire-fighters would want to try and set a counter fire. This section has a larger in-draft velocity than the flame-affected zone and also has no large recirculation patterns. This zone tails off as it moves away from the fire and drops off back to near atmospheric conditions dominate by the wind. In some of the simulations, which have high intensities and lower wind velocities, the drop-off length becomes constant at the end of the domain. This is likely to mean that the true drop-off point is outside the current computational domain. It was also found that for simulations with very low intensities and very high wind velocities the shape of the profile is not the same as Fig 1. This is because for strong wind velocities and weak bouncy-induced currents, the profile tends to flatten out and lose its some features. When the wind becomes the dominant current downstream the fire, counter-fire operations are not recommended. Each fire intensity has a maximum wind limit above which the peak in-draft zone is no longer present and thus the use of counterfires should not be recommended.

\subsection{The flame-affected zone}

The length of the flame-affected zone sets how close a counter-fire should be set to the main wildfire. Fig. 3 shows the dependence of $\mathrm{L}_{1}$ with the fire intensity over an average of the wind velocities. It was found that for fire intensities below $6 \mathrm{MWm}^{-1}, \mathrm{~L}_{1}$ increases but at intensities above this value it stays relatively constant around $28 \mathrm{~m}$. The in-draft wind velocities within this zone are in the range of 0 to $1 \mathrm{~ms}^{-1}$. The analysis of the dependence of $\mathrm{L}_{1}$ with the wind velocity for a range of fire intensities shows that in general terms the length increases with wind and that for high wind velocities it stays relative constant around 30 $\mathrm{m}$. At lower intensities the relationship becomes inconsistent and while the majority of the intensities led to reduced lengths for lower wind velocities, some of the higher intensity fire led to a relatively constant length around $30 \mathrm{~m}$ for all wind velocities.

\subsection{Wind dependent length near the flame $-L_{w}$}

As show in Fig. 4, for all intensities $\mathrm{L}_{\mathrm{w}}$ increases with increasing wind velocity. This is an expected result as the larger the wind velocity, the greater the slope angle the flame is slanted. 


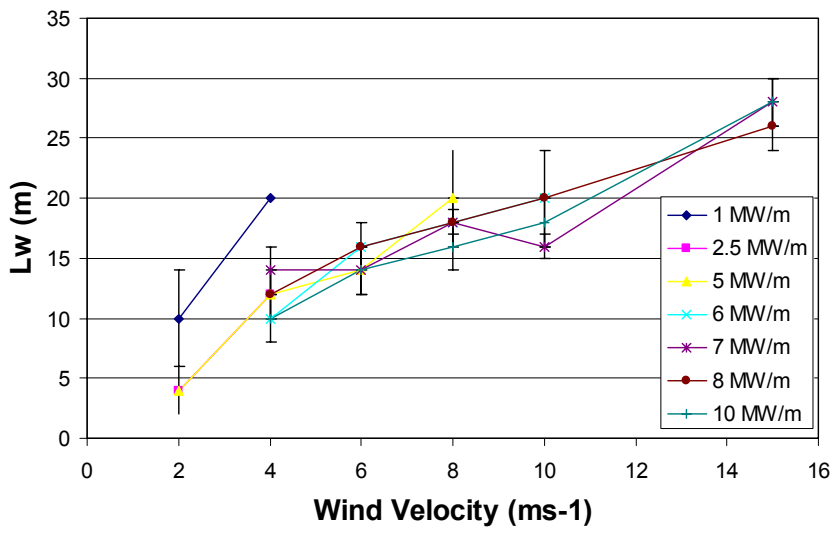

Figure 4: Wind dependent length near the flame vs. wind velocity for different fire intensities.

\subsection{The peak in-draft zone}

This zone is where the in-draft velocity profile is largest and thus the zone which has the most potential to be exploited when placing counter-fires. It is described by two important variables, its length ranging from 20 to $40 \mathrm{~m}$ and its average in-draft velocity ranging from 0.5 to $3.5 \mathrm{~ms}^{-1}$. The results of lengths of the zone vs. fire intensity shown in Fig 5 indicate that as the intensity of the fire is increased, so does the length of the section. This trend is produced by the large buoyancy-induced entrainment at the flame.

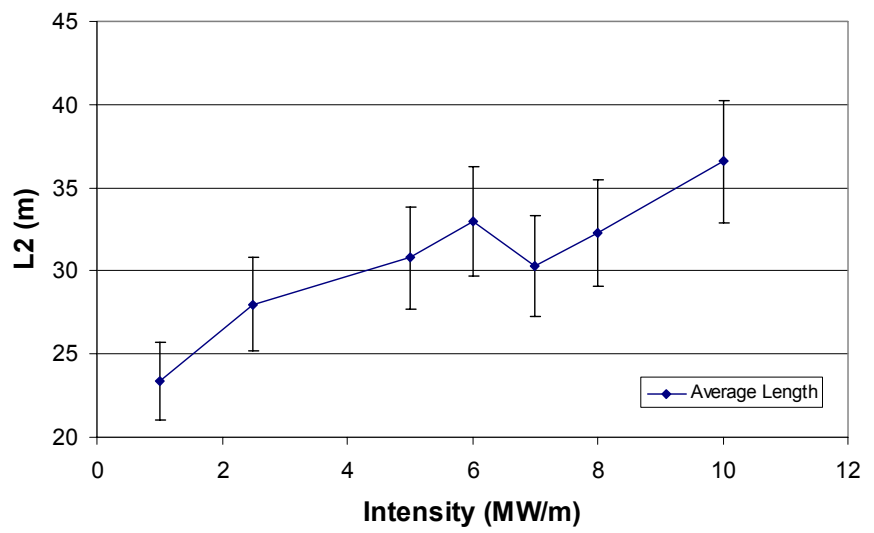

Figure 5: Average length of the peak in-draft zone against fire intensity averaged for a range of winds.

The dependence of $\mathrm{L}_{2}$ with the wind velocity for fire intensities above $1 \mathrm{MWm}^{-1}$ shows that the length decreases as the wind velocity increases up to 
$10 \mathrm{~ms}^{-1}$. This indicates that atmospheric wind tends to reduce the local in-draft velocity and reduce the range of this zone. For wind velocities over $10 \mathrm{~ms}^{-1}$ the length increases slightly. It is worth noting that at very high wind velocities (say above $10 \mathrm{~ms}^{-1}$ ), it is unlikely that counter fires will be effective as the wildfire front will spread at such fast rate that the resulting fire break will not be of enough width.

\subsubsection{Average in-draft velocity - $U_{a}$}

Perhaps more useful to counter fire operations is the average in-draft velocity $U_{a}$ located within the peak in-draft zone. This is a good global indicator of the indraft conditions. As the wind velocity increases the average velocity of decreases until at high winds it begins to level out and become constant around $1 \mathrm{~ms}^{-1}$. This indicates that the wind tends to reduce the in-draft towards the wildfire. Fig. 6 shows the dependence of $U_{a}$ with the fire intensity for different wind velocities. When the intensity of the fire is increased the average velocity increases. The greater intensity fires induce larger buoyant flow entrainment and hence large indraft at ground level. The range of values of the average velocity is lower than the peak as the average has to take into account the rise and fall from this peak. The average velocities are approximately half the magnitude of the peak velocities, ranging from $5.5 \mathrm{~ms}^{-1}$ to negligible velocities. The velocities have negative values to indicate the in-draft directed towards the wildfire and against the atmospheric wind.

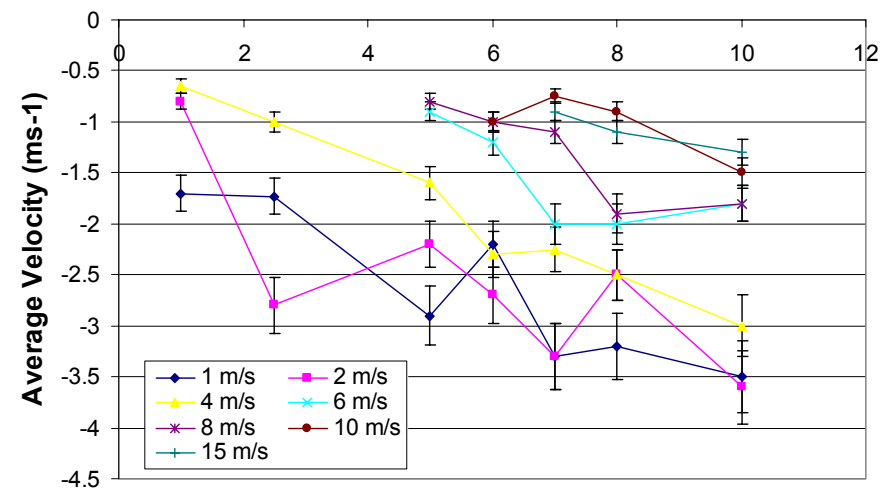

Fire Intensity (MW/m)

Figure 6: Average in-draft horizontal velocities in peak in-draft zone vs. fire intensity and wind.

\subsubsection{Drop-off zone}

The drop-off zone observed in the in-draft velocity profile (Fig. 1) marks the maximum distance away from a fire where a counter-fire can benefit from the indraft. If it is set beyond this length, it will not be subjected to the in-draft of the main wildfire and thus would simply be a secondary fire, much like a spot fire. This is therefore an important length and contains very useful information for 
fire-fighters. Fig. 7 shows the dependence of the drop-off length with the fire intensity for different wind velocities. It can be seen that for the lower wind velocities, under $6 \mathrm{~ms}^{-1}$, the length rises as the intensity increases but then reaches the end of the domain and becomes constant at around $60 \mathrm{~m}$. For wind velocities higher than $6 \mathrm{~ms}^{-1}$, the drop-off length becomes constant at around 50 $\mathrm{m}$ over the range of fire intensities. This implies that at high wind velocities the drop-off length is dictated by the wind velocity rather than the intensity.

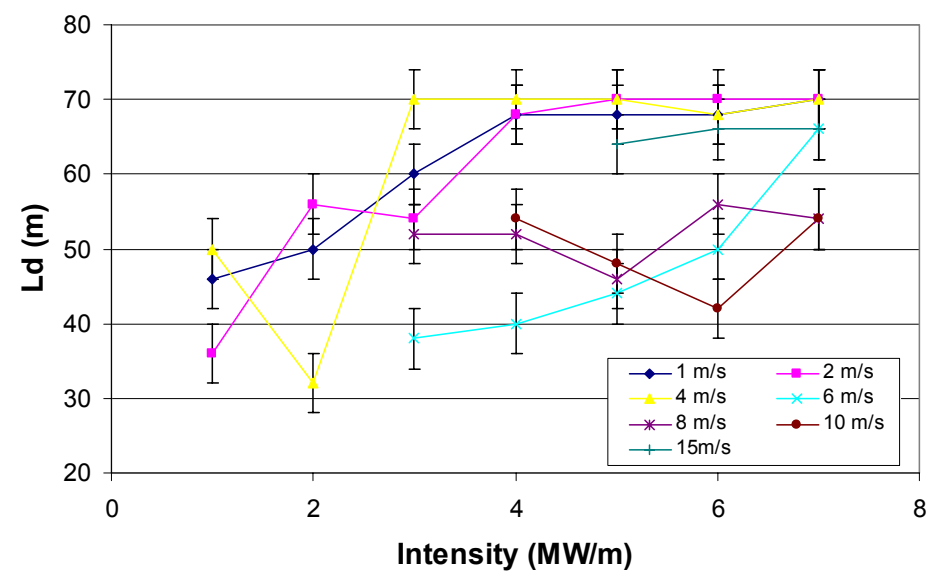

Figure 7: Drop-off length against fire intensity for different wind velocities.

Fig. 8 shows the drop-off length against the wind velocity for different fire intensities. Again, it can be seen that for the lower wind velocities the general trend is a decrease with increasing wind velocity below $10 \mathrm{~ms}^{-1}$. Above this wind velocity, the length rises slightly.

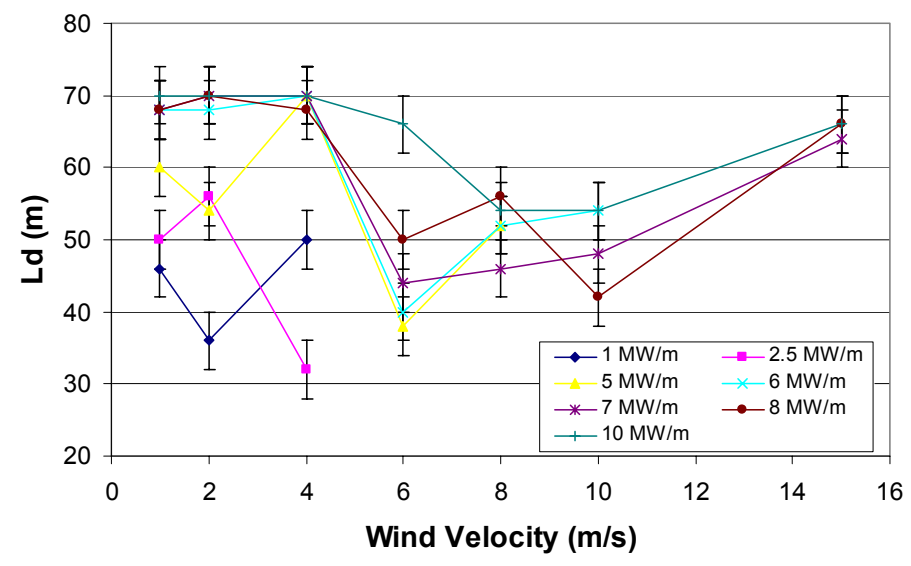

Figure 8: Drop-off length against fire intensity for different wind velocities. 


\section{Conclusions}

This paper has looked into the entrainment flow field downstream of wildfires of various intensities and subjected to different wind velocities. These computer simulations offer a phenomenological approximation to the problem. Three zones can be differentiated in the in-draft flow structure. The wind dependent part of flame-affected zone increases in length as the wind velocity is increased. Changes in the fire intensity where found to have little or no effect on it. The most suitable zone for pulling the counter-fire, the peak in-draft zone, has a characteristic in-draft at ground level around $2 \mathrm{~ms}^{-1}$ and is located between 15 and $70 \mathrm{~m}$ downwind from the flames, depending on the conditions. The effect of the fire intensity is to increase this average in-draft and the wind is to decrease it. The maximum distance that a counter-fire should be set from the wildfire, named the drop-off length, was found to increase with increasing intensity but decrease with increasing wind velocity. It is anticipated that this information can aid firefighting operations. This work is a first approximation to the problem and aims at providing fire-fighters an estimation of the range of in-drafts and distances to set counter-fires. However, the theoretical work remains approximate until field studies are conducted to validate these results.

\section{References}

[1] Snow SB, Clarke B, 1953, Forest Fire Control, FAO USA.

[2] Alexander ME, 2000, Forest Research, Wellington. Forest Research Bulletin No. 197, Forest and Rural Fire Scientific, No. 5. p. 30.

[3] Pyne SJ, 1984, Introduction to Wildland Fires: Management in the United States, Wiley and Interscience Publications

[4] Hostikka S, Mangs J, Mikkola E, 2008, Comparison of two and three dimensional simulations of fires at wildland urban interface, $9^{\text {th }}$ IAFSS

[5] Teie WC, Wildland Firefighting Fundamentals, Deer Valley Press, California, 2000.

[6] McGrattan K, Klein B, Hostikka S, Floyd J, 2007, Fire Dynamic Simulator (Version 5) User's Guide, NIST Special Publication 1019-5.

[7] Heskestad G, 2002, Fire Plumes, Flame Height and Air Entrainment, The SFPE Handbook of Fire Protection Engineering, $3^{\text {rd }}$ Ed. 